\title{
Reduction of corneal scarring in rabbits by targeting the TGFB1 pathway with a triple siRNA combination
}

\author{
Sriniwas Sriram ${ }^{1}$, Daniel Gibson ${ }^{2}$, Paulette Robinson ${ }^{2}$, Sonal Tuli ${ }^{3}$, Alfred S. Lewin ${ }^{4}$, Gregory Schultz $^{1}$ \\ ${ }^{1}$ Department of Biomedical Engineering, University of Florida, Gainesville, USA \\ ${ }^{2}$ Institute for Wound Research, Department of Obstetrics and Gynecology, University of Florida, Gainesville, USA \\ ${ }^{3}$ Department of Ophthalmology, University of Florida, Gainesville, USA \\ ${ }^{4}$ Department of Molecular Genetics and Microbiology, University of Florida, Gainesville, USA \\ Email: vass87@gmail.com
}

Received 30 August 2013; revised 19 September 2013; accepted 5 October 2013

Copyright (C) 2013 Sriniwas Sriram et al. This is an open access article distributed under the Creative Commons Attribution License, which permits unrestricted use, distribution, and reproduction in any medium, provided the original work is properly cited.

\section{ABSTRACT}

Purpose: The transforming growth factor beta1 (TGFB1) pathway has been linked to fibrosis in several tissues including skin, liver, kidney and the cornea. In this study, a RNA interference-based approach using siRNAs targeting three critical scarring genes, TGFB1, TGFB receptor 2 (TGFBR2) and connective tissue growth factor (CTGF), was tested for effects on reducing alpha smooth muscle actin (SMA) and corneal scarring (haze) in excimer laser ablated rabbit corneas. Methods: Levels of TGFB1 and CTGF mRNAs were measured using qRT-PCR in the epithelial and endothelial cell layers of normal and excimer ablated rabbit corneas at 30 minutes, 1 day and 2 days after ablation. Two different scarring models were utilized to assess the effects of the triple siRNA combination on corneal scarring. In the first model, rabbit corneas were unevenly ablated creating a mesh pattern then treated immediately with the triple siRNA combination. After 1 day the ablated areas of corneas were collected and levels of mRNAs for TGFB1, TGFBR2 and CTGF were measured. After 14 days, levels of mRNA for SMA were measured and SMA protein immunolocalized in frozen sections. In the second model, rabbit corneas were uniformly ablated to a depth of 155 microns followed by three daily doses of the triple combination of siRNA. After 14 days, corneas were photographed and images were analyzed using Image $\mathrm{J}$ software to assess corneal scarring. Corneas were also analyzed for levels of SMA mRNA. Results: In both unwounded and wounded corneas, levels of TGFB1 and CTGF mRNA were always significantly higher in endothelial cells than in epithelial cells (10 to 30 fold). Thirty minutes after injury, levels of both TGFB1 and CTGF mRNAs increased approximately 20-fold in both epithelial and endothelial cells, and further increased approximately 60 -fold in 2 days. In the first therapeutic experiment with a single siRNA dose, two of three rabbits showed substantial reductions of all three target genes after 1 day with a maximum knock down of $80 \%$ of TGFb1, $50 \%$ reduction of TGFBR2 and $40 \%$ reduction of CTGF mRNA levels and reduced SMA mRNA at day 14. In the second therapeutic experiment with multiple doses of siRNA treatment, both rabbits showed a $\sim 22 \%$ reduction in scar formation at day 14 as calculated by image analysis. There was also a corresponding $70 \%$ and $60 \%$ reduction of SMA RNA expression. Conclusion: These results demonstrate that both TGFB1 and CTGF dramatically increase in rabbit corneal epithelial and endothelial cells after injury. Treatment of excimer ablated rabbit corneas with a triple combination of siRNAs effectively reduced levels of the target genes and SMA, leading to reduced corneal scarring at 14 days, suggesting that this triple siRNA combination may be an effective new approach to reducing scarring in cornea and other tissues.

Keywords: RNA Interference; siRNA Combination; Corneal Scarring; TGFB1; CTGF

\section{INTRODUCTION}

Corneal scarring remains a serious complication that can ultimately lead to functional vision loss. In an injured cornea, a cascade of molecular events is initiated by prolonged, elevated levels of transforming growth factor beta (TGFB1) which then combines with the Transforming Growth Factor Receptor II (TGFBR2) inducing the synthesis of Connective Tissue Growth Factor (CTGF) causing excessive scarring (corneal haze) that impairs vi- 
sion. The TGF- $\beta$ system, has emerged as a key component of the fibrogenic response to wounding by regulating the transformation of quiescent corneal keratocytes into activated fibroblasts that synthesize ECM and into myofibroblasts that contract corneal matrix (Chen et al. 2000; Jester, Petroll, and Cavanagh 1999). These myofibroblasts are filled with alpha smooth muscle actin (SMA) that forms microfilaments that are the major source of light scattering in corneal scars [1]. CTGF acting as a downstream mediator of TGFB1, down regulates synthesis of corneal crystallin proteins in quiescent keratocytes and up regulates synthesis of collagen. Thus, the excessive scattering of light that is clinically described as corneal scar and haze results from the combination of collagen laid down in irregular pattern in the wound and opaque activated fibroblasts and myofibroblasts that no longer synthesize the corneal crystallin proteins that keep their cytoplasm transparent.

We have previously shown the effect of PRK on CTGF levels in rat and mouse corneas and found that CTGF was present in all cell layers of the cornea. The levels of CTGF were found to continually rise from the time of wounding up through 28 days post wounding [1]. However, in order to completely understand the role of TGFB1 in tissues repair and scarring, it is essential to understand the timing and site of the synthesis of these growth factors so that the appropriate cell layer is targeted for nucleic acid therapies. The experiments in this study are expected to reveal when and where TGFB1 and CTGF are synthesized after a corneal injury so that the best mode of action for an anti-fibrotic therapy can be chosen.

There currently are no FDA approved drugs that selectively reduce the expression of genes causing corneal scarring and haze. At present, the methods used to decrease corneal haze are topically applied steroids or antimetabolite drugs that target the cells capacity to respond to signaling. Mitomycin $\mathrm{C}$ is used during some ocular surgeries, but it may have very damaging side effects, such as epithelial defects, stromal melting, endothelial damage, and conjunctival thinning [2]. Hence, there is a need to develop a targeted approach that can nullify the specific molecular pathways that give rise to a scar.

It is however difficult to achieve significant therapeutic effect by employing a one-target, one-drug paradigm on such a complex, multi-factorial signaling pathway. Hence, using a multi-target approach can interrupt or act on the complex signaling network at multiple points, and affect the cell in ways that an individual component cannot [3]. In this study, we have tested a siRNA triple combination targeting TGFB1, TGFBR2 and CTGF. This triple siRNA combination was shown to be effective in reducing the expression of target (TGFB1, TGFBR2 and CTGF) and downstream mediators like Collagen-I and $\alpha$-Smooth Muscle Actin (SMA) in both in vitro cell cul- ture system and ex vivo organ cultures [4].

Additionally, it is important to deliver these siRNA combinations to the corneal layer where there is high localization of the target growth factors after wounding. Although most of the targeted anti-fibrotic approaches target the stromal fibroblasts due to the eventual presence of myofibroblasts in this region, there has not been any research on the post-wounding localization ofTGFB1 and CTGF in the epithelium and the endothelium. A delivery method that targets the corneal layer with maximum postwounding growth factor localization is critical for an effective therapy.

Delivery of drugs to the cornea is a major challenge, as the mechanical barriers that protect the cornea (multilayered epithelium, tight junctions) constrain ocular drug delivery [5]. The principal properties governing corneal drug absorption are its lipophilicity, partition coefficient and molecular size [6]. Nanocarriers are the potential solution for targeted ocular drug delivery as they have been shown to be non-immunogenic, have relatively low toxicity, be resistant to protein/serum absorption and be stable in an enzymatic environment [7]. We have previously showed the high efficacy of the nanoparticle kit used in this study in delivering fluorescently labeled siRNA to all layers of the cornea including the endothelium in an $e x$ vivo organ culture model [8].

The overall goal of this study is to test and deliver a previously optimized effective triple siRNA combination to the appropriate corneal layer with high post wounding localization of TGFB1 and CTGFso that there is a maximal reduction of scar formation in rabbits.

\section{METHODS}

\subsection{Laser Ablation of Rabbits}

Adult New Zealand Rabbits free of disease were used and treated according to ARVO Statement for the Use of Animals in Ophthalmic and Vision Research. Excimer ablation and collection of corneas was performed as previously described [9]. Briefly, rabbits were anesthetized with isoflurane inhalation, and proparacaine eye drops provided topical anesthesia. Laser ablations were performed to both eyes of each rabbit with a Summit SVS exicimer laser that is committed to animal vision research. In this study, two different approaches were tested to obtain the most intense scarring in the rabbits. In the first approach, using the laser in phototherapeutic keratectomy mode, the central $6 \mathrm{~mm}$ diameter area of the cornea was ablated at a dose of $160 \mathrm{~mJ} / \mathrm{cm}^{2}$ to an initial depth of 80 microns to remove the epithelium and then the final 45 microns were ablated by placing a mesh over the cornea to make an uneven ablation. In the second approach, using the same laser parameters, the central $6 \mathrm{~mm}$ diameter area of the cornea was ablated to an even depth of 155 
microns.

The eyes were then pretreated with $50 \mu \mathrm{M}$ EDTA for 10 minutes. A total of $150 \mu$ of the nanoparticle complexed with the siRNA triple combination was added to one of the eyes while the other was treated with the vehicle control and was considered as a paired negative control. The eyes were held open for 3 minutes to allow the nanoparticle to penetrate the stroma before being disturbed. No postoperative topical steroid was used to ensure that the wound healing process is not altered with anti-inflammatory agents. Corneas were collected at different time points according to the experiment, homogenized in a pestle with liquid nitrogen and then transferred to TRIzol. The RNA was then extracted using a hybrid RNA extraction protocol with RNeasy spin columns [10].

\subsection{Gross Corneal Dissection}

Rabbits without observable corneal wounds were anesthetized, excimer ablated to 125 microns and euthanized at the designated time points as described before. A scalpel was used to immediately scrape the epithelium off with care taken to ensure that the scraped mass was retained on the blade. The scraped epithelial mass was then transferred to $350 \mu \mathrm{l}$ of tissue lysis buffer (Qiagen, buffer RLT) and the blade was rinsed with $250 \mu$ of additional lysis buffer. The cornea was then excised from the globe by cutting with a fresh scalpel and scissors at the corneal/ scleral boundary. The cornea was placed face down and yet another fresh scalpel was used to scrape off and retain the endothelium as was done with the epithelium. The endothelial mass was transferred to $350 \mu \mathrm{l}$ of lysis buffer and the blade rinsed with an additional $250 \mu \mathrm{l}$ of lysis buffer. Each grossly isolated cellular layer was then subjected to ultrasonication on ice for further tissue disruption. The probe was rigorously washed, rinsed and dried in between each sample. The homogenates were then immediately loaded onto Qiagen gDNA removal columns and the RNA was purified in accordance with the manufacturer's provided protocol (Qiagen RNA easy, Qiagen, Inc., Cat. \#74104).

The purified RNA was quantified via ultra-violet absorbance using a Nanodrop ND-1000 spectrophotometer set for RNA quantification.

\subsection{Preparation of siRNA-Nanoparticles}

A commercially available nanoparticle kit called Invivoplex ${ }^{\circledR}$ was purchased from Aparnabio (Rockville, MD) and used according to manufacture's instructions. Briefly, siRNA triple combination solution was made at a concentration of $0.9 \mathrm{mg} / \mathrm{ml} .600 \mu \mathrm{L}$ of this solution was added with $300 \mu \mathrm{L}$ of the provided cargo buffer. This solution was added drop wise to $900 \mu \mathrm{L}$ of the given nanoparticles over a magnetic stirrer. This preparation forms
siRNA nanoparticles $<50 \mathrm{~nm}$ that are stable for a week.

\subsection{Reverse Transcription-Polymerase Chain Reaction}

Total RNA was extracted using the Qiagen RNeasy mini isolation kit (Qiagen, Inc., Valencia, CA) and used according to the manufacturer's directions. cDNA was synthesized using the High Capacity cDNA Reverse Transcription Kit (Applied Biosytems, Carlsbad, CA) according to manufacturer's procedure. The level of mRNA for TGFB1, CTGF and SMA were determined using the RealTime PCR TaqMan assay. The primers and probes for each gene are defined in Table A1. The endogenous control, ribosoma18S RNA and GAPDH was used normalize target genes. Primers, probes and cDNA were combined with TaqMan Universal PCR Master Mix (Applied Biosytems, Carlsbad, CA) and amplification was performed by the Applied Biosystems 7300 HT Fast Real Time PCR System (Carlsbad, CA). A few samples were run without reverse transcriptase to measure the quantity of genomic DNA (gDNA) present in the sample. The thermal cycling conditions were as follows: $2 \mathrm{~min}$ at $50^{\circ} \mathrm{C}, 10 \mathrm{~min}$ at $95^{\circ} \mathrm{C}, 40$ cycles of $15 \mathrm{sec}$ at $95^{\circ} \mathrm{C}$, and $1 \mathrm{~min}$ at $60^{\circ} \mathrm{C}$. The relative gene expression of the growth factors was calculated using the 2- $\Delta \Delta \mathrm{Ct}$ method.

\subsection{Macrophotography}

Prior to general anesthesia, each eye was topically anesthetized with proparacaine and each pupil was dilated with phenylephrine $2.5 \%$ and tropicamide eye drops. Each rabbit was then generally anesthetized with inhaled isoflurane as described earlier. The eyelids were held open and out of the way with either an eyelid speculum or a pair of cotton swabs. A Nikon D7000, was outfitted with a macro lens capable of native 1:1 reproduction (either a $100 \mathrm{~mm}$ Tokina or $60 \mathrm{~mm}$ Nikkor) and the Nikon R1C1 Creative Lighting System (CLS) flash system. The D40 was set to the "Normal" program, ISO 200, manual exposure with a shutter speed of 1/500 second and f/16. While the 7000 was set to the "Standard" program, ISO 100 , manual exposure with a shutter speed of $1 / 250 \mathrm{sec}-$ ond and $\mathrm{f} / 18$. To visualize and measure haze, the flash power was set manually (1/16th, D40, 1/6.4th D7000) and neither the flash nor lens had a filter. For all images, the lens was set to manual focus and pre-focused to a 1:1 reproduction ratio and the camera was focused by moving the camera closer or further from the subject. Guide lights on the flash heads were used to facilitate haze visualization and focusing.

\subsection{Immunohistochemistry}

The rabbit corneas from the experiments were fixed overnight in $4 \%$ paraformaldehyde. They were then bi- 
sected, fixed in OCT and then sectioned in $10 \mu \mathrm{m}$ slides. The slides were then washed with PBS and blocked in horse serum for 1 hour. Finally, they were incubated with SMA antibody-Cy3 (Sigma) for 1 hour at room temperature. The slides were mounted with DAPI and imaged using fluorescence microscope.

\subsection{Statistical Analysis}

All experiments were performed in triplicate and all statistical analyses were conducted using Graph Pad prism (San Diego, CA). Student's t test or Analyses of Variances (ANOVA) with Tukey's post-hoc assessments were accordingly used to test for significance between the groups. Results were considered statistically significant where $\mathrm{p}<0.05$.

\section{RESULTS}

The levels of TGFB1 and CTGF were analyzed at several time points following ablation to find the best time to dose with anti-scarring drugs. Two different models of scarring were tested to find which of the two generated the most intense scaring in rabbits. Also in this study, the in vivo efficacy of a previously optimized triple siRNA combination that was effective in reducing downstream scarring genes (SMA and collagen-I) in both in vitro culture and ex vivo organ cultures was evaluated. The triple siRNA combination targets three critical scarring genes

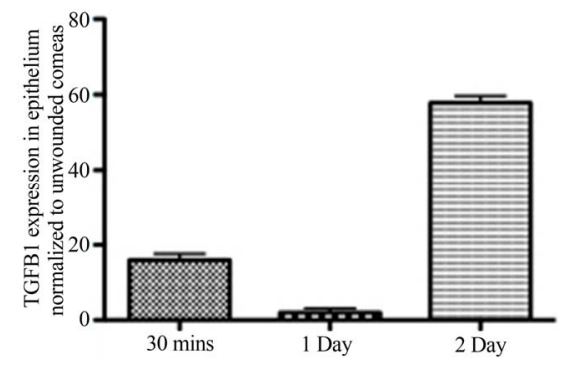

(a) within the same TGFB1 pathway-TGB1, TGFBR2 and CTGF $[4,8]$.

\subsection{The Effect of PRK on mRNA Levels of TGFB1 and CTGF}

The corneas of 11 rabbits were evenly ablated to $125 \mathrm{mi}-$ crons using an excimer laser. 3 rabbits were sacrificed at 30 mins after ablation, and 4 rabbits each for Day1 and Day2 post-ablation. The corneas of three rabbits were unablated and used as control. At the designated time points, the corneas were collected and the epithelium and endothelial layers were scrapped using a surgical scalpel and collected in separate tubes. The expression levels of TGFB1 and CTGF were analyzed using qRT PCR. As shown in Figure 1, there is an initial spike in the levels of TGFB1 and CTGF as early as 30 minutes after ablation. There is then a decrease in the expressions at Day 1 followed by an exponential increase on Day2. The expression trend of TGFB1 and CTGF follows a similar trend in both the epithelial and endothelial layers. Figure 1(e) plots the RNA level expressions of TGFB1 and CTGF in terms of the epithelium. The expressions of both TGFB1 and CTGF were consistently higher in the endothelial layer when compared to the epithelium particularly at Day1 when the expression of CTGF in endothelium was $~ 35$ times that of the epithelium. All expressions were calculated with respect to the unablated corneas and were nor-

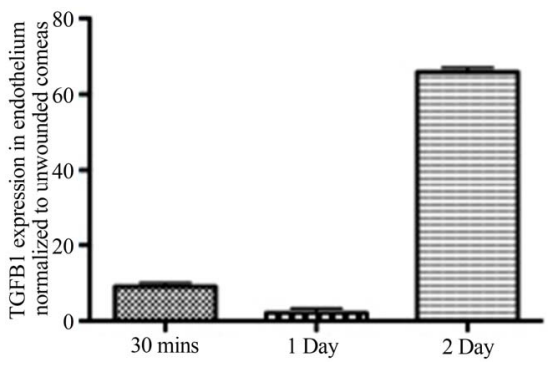

(b)

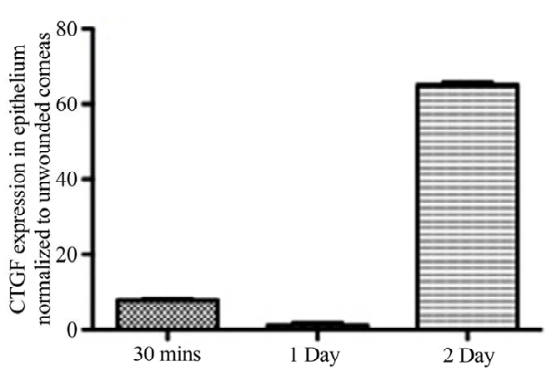

(c)

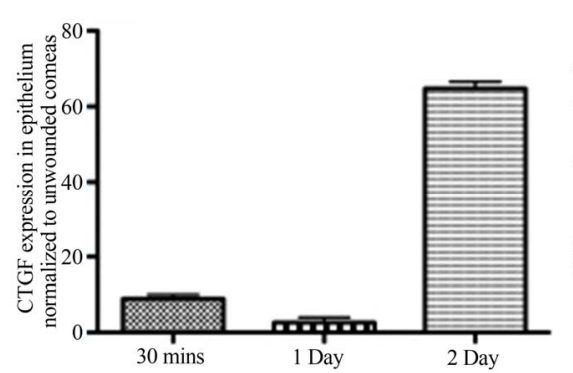

(d)

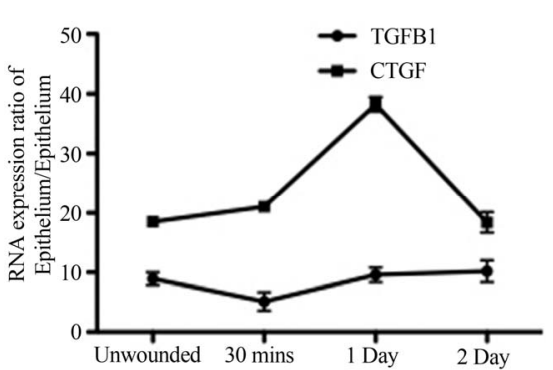

(e)

Figure 1. Post-ablation expression timeline of TGFB1 and CTGF. The corneas of 11 rabbits were evenly ablated to 125 microns using an excimer laser. 3 rabbits were sacrificed at 30 mins after ablation, and 4 rabbits each for Day 1 and Day 2 post-ablation. The corneas of three rabbits were unablated and were used as the control. The corneas were collected and the epithelium and endothelial layers were scrapped using a scalpel and collected in separate tubes. The expression levels of TGFB1 and CTGF were analyzed using qRT PCR. All expressions were calculated with respect to the unablated corneas and was normalized using GAPDH as the housekeeping gene. Figure (e) plots the RNA expressionsof TGFB1 and CTGF in endothelium in terms of the epithelium. 
malized using GAPDH as the housekeeping gene.

\subsection{The Effective Triple Combination (T1R2C1) Inhibits Target mRNA Accumulation in Rabbits}

The corneas of 9 rabbits were unevenly ablated to 125 microns using an excimer laser. The right eye was treated with $150 \mu \mathrm{L}$ of the effective triple combination (T1R2C1) complexed with nanoparticles and the left eye received equal volume of the vehicle control. One day later, 3 rabbits were humanely sacrificed and total RNA was extracted for analysis by RT-PCR. The effective triple combination (T1R2C1) gave an average of knockdown of $57 \%$ for TGFB1, 25\% for TGFBR2 and 24\% for CTGF (Figure 2). One of the rabbits (rabbit 1) had a maximum knockdown of $80 \%$ for TGFB1, 57\% for TGFBR2 and $46 \%$ for CTGF indicating some the siRNA combination was effectively delivered to the corneal stroma in this animal. The knockdown percentages were calculated with respect to the left eye, which received vehicle control without the siRNA.

\subsection{SMA Immunohistostaining in Triple siRNA Treated Rabbit Corneas}

6 out of the 9 treated rabbits from the above experiment were used for a long-term experiment to observe scar formation. After 14 days, the intensity of scarring in both the treated and the control eyes was graded by a masked ophthalmologist and was also imaged using a digital camera. The rabbits were then humanely sacrificed and three cor-

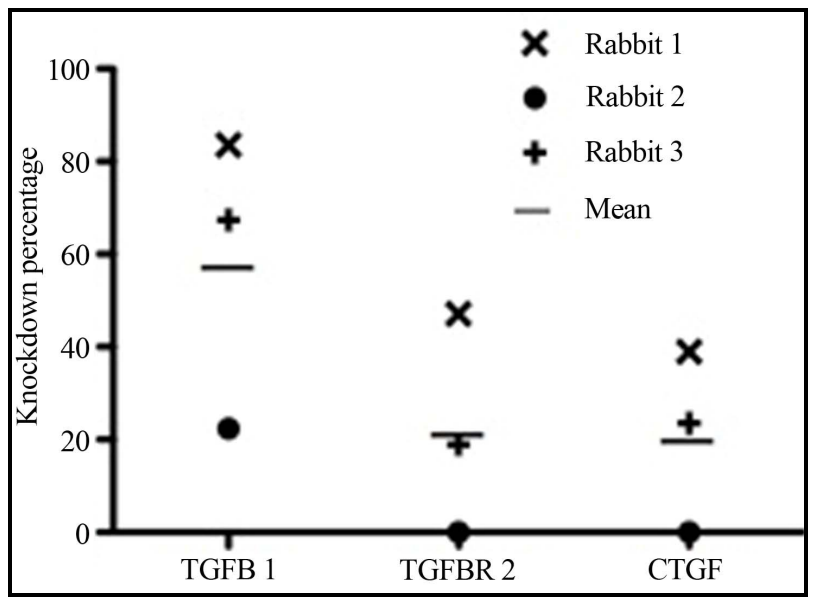

Figure 2. Short-term knockdown of target growth factors. The corneas of 3 rabbits were unevenly ablated to 125 microns using an excimer laser. The right eye was treated with the effective triple combination (T1R2C1) complexed with nanoparticles and the left eye received the vehicle control. One day later, the rabbits were sacrificed and RNA was extracted for analysis by qRT PCR. The figure gives the RNA level knockdown percentages of the target growth factors calculated with respect to the left eye. All expressions were normalized to $18 \mathrm{~S}$ rRNA. neas were collected for SMA immunohistostaining. The corneas were fixed overnight in $4 \%$ paraformaldehyde. They were then bisected, fixed in OCT, sectioned in 10 $\mu \mathrm{m}$ slides and stained for SMA. The treated cornea of two of the three rabbits selected for immunohistostaining had a lower haze grading score when compared to the control cornea. The control eye that was ablated and treated with vehicle control shows SMA staining in the basal epithelium and stroma while the triple siRNA treated right eye shows reduction in SMA staining (Figures 3(b) and (d)).

Three corneas from the 6 treated rabbits from the above experiment were collected for RNA level analysis by qRT PCR. SMA knockdown percentage of $>40 \%$ was observed in 2 out of the 3 rabbits. The untreated left eye in these rabbits had haze-grading scores of 2 and 3 respectively. No knockdown was observed in the other rabbit, which had a haze grading score of one in the untreated left eye. The RNA level knockdown percentage of SMA was calculated with respect to the untreated left eye and all expressions were normalized to $18 \mathrm{~S}$ rRNA.

\subsection{Macrophotography Images of Reduction in Scarring by Repeated siRNA Dosing}

The corneas of 3 rabbits were evenly ablated to $155 \mathrm{mi}$ crons using an excimer laser. One of the rabbits had a pre-existing scar and spots of neovascularization on the

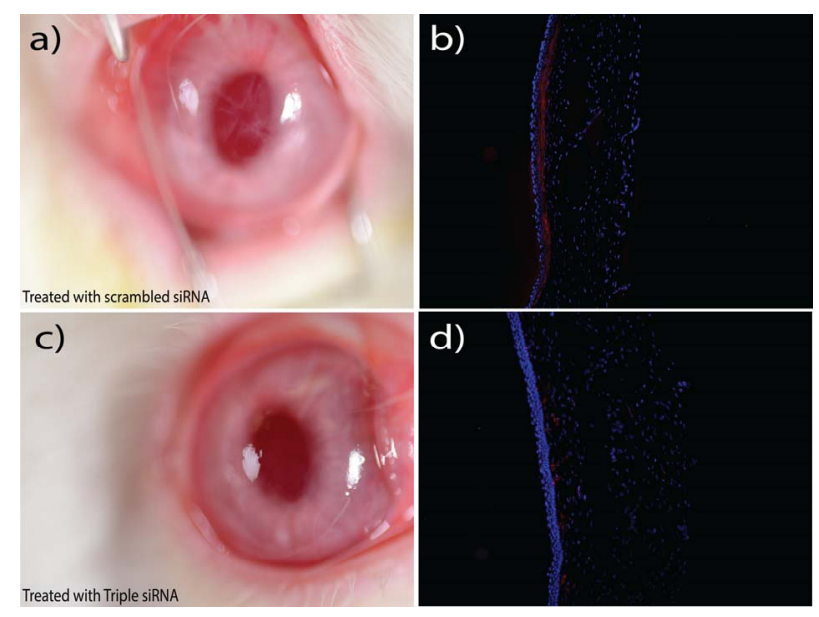

Figure 3. SMA immunohistostaining in triple siRNA treated rabbit corneas. The corneas of 6 rabbits were unevenly ablated to 125 microns using an excimer laser. The right eye was treated with the effective triple combination (T1R2C1) complexed with nanoparticles and the left eye received the vehicle control. 14 days later, the rabbits were sacrificed and three corneas were collected for SMA immunohistostaining. The corneas were fixed overnight in $4 \%$ paraformaldehyde. They were then bisected, fixed in OCT and then sectioned in $10 \mu \mathrm{m}$ slides. To stain for SMA, slides were blocked in horse serum and then incubated with cy3 labeled SMA antibody (red). The control eye that was ablated and treated with vehicle control shows SMA staining in the basal epithelium and stroma while the triple siRNA treated right eye show reduction in SMA staining. 
right eye and hence had to be excluded. The right eye was treated with the effective triple combination (T1R2C1) complexed with nanoparticles for the first three days after ablation while the left eye was left untreated. After 14 days, the corneas of both rabbits were imaged using the macrophotography technique described in the methods section. The haze grading scores of both the rabbits were similar with Rabbit b showing a slight reduction in scarring after treatment (Figure 4).

\subsection{Quantification of Scar Reduction by the siRNA Treatment}

The digital images from the above experiment were subjected to anti-red gray scale conversion by only using the data in the blue channel. The contrast was increased by automatic brightness correction in Image J. The wounding region was split into two regions-Top (Region-I) and Bottom (Region-II) (Figures 5(a) and (b)). The pixel intensities of the regions of interest were normalized to that of the transparent unwounded regions of the corresponding corneas. The percentage of haze reduction was calculated by the reduction in pixel intensity of the scarring region in the treated eye with respect to that of the untreated eye. The region-I of both the rabbits show an average of $\sim 22 \%$ reduction in scarring due to the siRNA treatment (Figure 5(c)). However there was no visible reduction in scar formation in region II of both the rabbits.

After imaging, the wounding region in the corneal tissues was collected with an 8-mm biopsy punch for RNA analysis. In the corneas treated with the triple siRNA combination, both the rabbits show a corresponding reduction of $60 \%$ and $40 \%$ in the RNA level expression of SMA

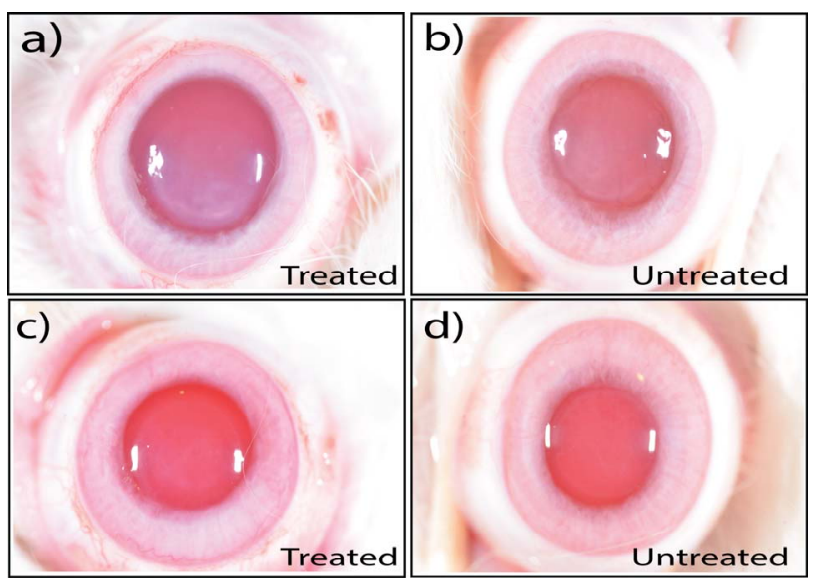

Figure 4. Reduction in scarring by repeated siRNA dosing. The corneas of 3 rabbits were evenly ablated to 155 microns using an excimer laser. The right eye was treated with the effective triple combination (T1R2C1) complexed with nanoparticles for the first three days after ablation while the left eye was left untreated. Both eyes were imaged using a digital camera after 14 days.
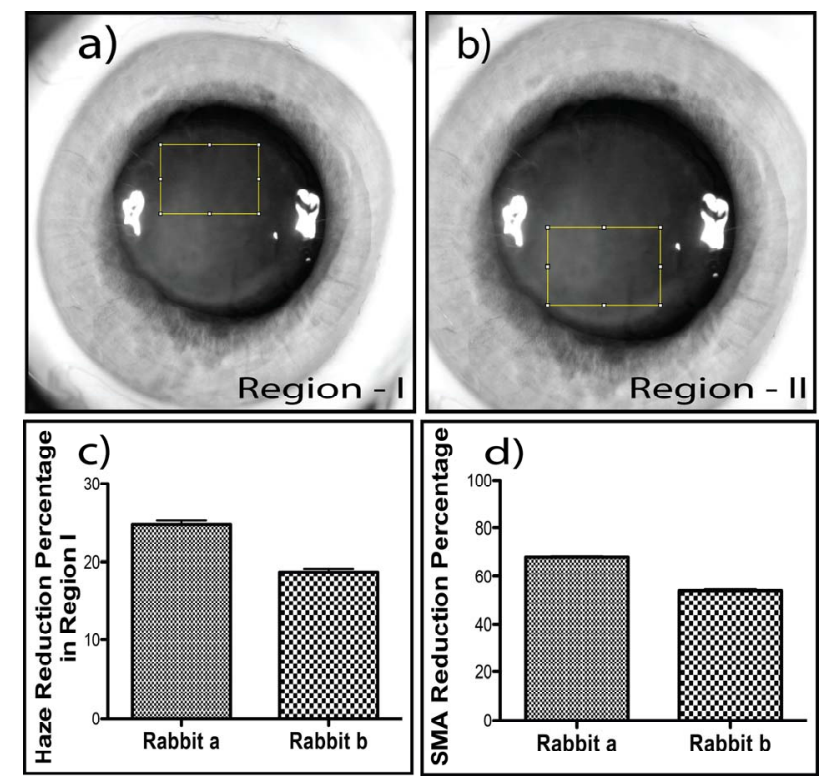

Figure 5. Quantification of scar reduction. The digital images from the above experiment were subjected to anti-red grayscale conversion by using the data in the blue channel. The contrast was increased by automatic brightness correction in ImageJ. The wounding region was split into two regions-Top (Region-I) and Bottom (Region-II). The pixel intensities of the regions of interest were normalized to that of the transparent unwounded regions of the corresponding corneas. The percentage of haze reduction was calculated by the reduction in pixel intensity of the scarring region in the treated eye with respect to that of the untreated eye (Figure (c)). After imaging, the wounding region in the corneal tissues was collected with a 6-mm biopsy punch for RNA analysis. Figure (d) gives the RNA level knockdown percentage of SMA in the corneas calculated with respect to the untreated eye. All expressions were normalized to $18 \mathrm{~S}$ rRNA.

(Figure 5(d)). The knockdown percentages of SMA in the corneas were calculated with respect to the untreated eye. All expressions were normalized to $18 \mathrm{~S}$ rRNA.

\section{DISCUSSION}

Several papers discuss the importance of the TGFB1 pathway acting through CTGF to activate quiescent corneal keratocytes and transform them into fibroblasts that synthesize collagen and further transform into myofibroblasts [11-13]. However, there has been very little research on which cell layers of the cornea synthesize high levels of TGFB1 and CTGF. Our observation that both TGFB1 and CTGF mRNA levels are the highest in the corneal endothelium is novel, and it has reshaped the thinking behind the primary target cells and future delivery methods for corneal anti-fibrotic therapies. The immediate increase in the expressions of TGFB1 and CTGF as early as 30 minutes post ablation highlights also emphasized the importance of an immediate treatment. The nanoparticle delivery system used in this study was able to successfully 
deliver siRNA to all layers of the cornea including the endothelium in an ex vivo organ culture model [8].

The results of the initial therapeutic experiment assessing the efficacy of a single application of the triple siRNA combination demonstrated the "proof of principle" that the siRNAs could be effectively delivered into the target corneal cells. However, there was variability in the level of knockdown among the three rabbits, suggesting that the dosing was not optimized for in vivo experiments. Factors that could contribute to the variability include the presence of a nictitating membrane in addition to the eyelids and tears that tend to clear the surface of the cornea and reduce drug exposure time. It is also possible that the volume of the siRNA formulation used for the single dose $(150 \mu \mathrm{L})$ in this experiment may also have been too high, which would have reduced the amount of the siRNAs that penetrated the cornea and were instead washed away once the trephine was removed.

The reduction in scar formation, 14 days after a single dose of triple siRNA treatment, also showed a positive trend. Three out of six rabbits had a reduction in scar formation as observed in the haze grading scores of a masked ophthalmologist. Both the digital image and the immunohistostaining staining for SMA in Figure $\mathbf{3}$ showed a reduction in scarring in the treated corneas when compared to the control. Both of these measurements were, however, qualitative, and we were unable to accurately quantify the exact reduction in scarring due to the lack of a standard imaging technology in the field of corneal scarring for those rabbits.

There was an interesting trend associated with the haze grading scores and the RNA knockdown percentage of SMA. We observed a RNA knockdown of $40 \%$ and $50 \%$ in the two rabbits that had a haze grading score of 2 and 3 respectively. However, no SMA knockdown was observed in the rabbit with a haze grading score of 1 . This suggests that the triple siRNA combination is effective in knocking down SMA expressions in intense scars and is less effective in case of mild scarring.

A key paper in the literature reports that deeper ablations during PRK lead to more intense scarring in animals [9]. In the second therapeutic experiment, we createda deep corneal ablation of 155 microns and also repeated the dosing of the triple siRNA combination for the first 3 days after ablation. Neither of the two rabbits in this experiment, however, developed an intense scar in the untreated control corneas. The haze grading scores of both the rabbits were similar with the one showing a slight reduction in scarring after siRNA treatment. However, the image analysis on the digital images revealed an average of $\sim 22 \%$ reduction in scarring in region 1 of both the corneas treated with the siRNA combination (Figure 5). It is interesting to note that the siRNA treatment had an effect in reducing scarring in Region 1, but not in Re- gion 2. This could be due to the uneven transfection of the siRNA-nanoparticle complex into the different corneal layers. The RNA level expression of SMA was also reduced in both the rabbits by $60 \%$ and $40 \%$, respectively.

In this study, we performed a series of pilot experiments to better understand the dynamics involved in the in vivo translation of an effective in vitro anti-fibrotic drug. The most immediate problem associated with the testing of an anti-fibrotic drug in the cornea of rabbits is the generation of a consistent and intense scar. We tried two different models of scarring in this study and although the corneas develop mild to average hazing, there is no consistent and intense scarring among animals. Since the variation in intensity of scarring among animals is natural and cannot be controlled, treating the corneas with TGFB1 post ablation for the first two days may help in the intensification of scar formation [14].

Another major hurdle in evaluating the efficacy of anti-fibrotic drugs in the cornea is the lack of a standard imaging technology to quantify the reduction in scarring. The current clinical method of haze grading by a masked ophthalmologist is qualitative and very subjective. Although the macrophotography technique described in this study is a lot more objective than haze grading, it also has its disadvantages. The dual flash heads create an unwanted reflection on the cornea due to which we are unable to measure the entire wounding region. A potential solution to this problem could be to image and analyze the corneas after excision. This would help in uniform illumination of the cornea without flash bias and the scar can also be measured as a function of the transparency.

Finally, the dosing regimen needs to be optimized so that maximum amount of inhibitory RNA can be delivered to the cornea. Lowering the volume and administering the siRNA cocktail for 2 - 3 days after the surgery along with an agent to increase the viscosity of the drug, so that it sticks to the surface of the cornea for a longer duration, might help increase the drug exposure time [15]. Other options could also include iontophoretically driving the drug to different layers of the cornea [16,17].

Although, the triple siRNA treatment in this study did not completely eliminate scarring, it did generate a strong positive trend in the reduction of scarring. It is important to understand and resolve the problems associated with the generation of intense scarring, drug delivery optimization and scar imaging before investing in a major in vivo experiment with many animals. Once these parameters are optimized, the triple siRNA combination could lead to a significant reduction of scarring in the cornea and perhaps also in other tissues.

\section{ACKNOWLEDGEMENTS}

Supported by Grants from the US Army Medical Research Acquisition 
Activity W81XWH-10-2-0917, National Eye Institute grant EY000587, National Eye Institute T32-EY07132 training grant, and the National Eye Institute P30-EY021721 Vision Core Grant.

\section{REFERENCES}

[1] Blalock, T.D., Duncan, M.R., Varela, J.C., Goldstein, M.H., Tuli, S.S., Grotendorst, G.R. and Schultz, G.S. (2003) Connective tissue growth factor expression and action in human corneal fibroblast cultures and rat corneas after photorefractive keratectomy. Investigative Ophthalmology \& Visual Science, 44, 1879-1887. http://dx.doi.org/10.1167/iovs.02-0860

[2] Azar, D.T. and Jain, S. (2001) Topical MMC for subepithelial fibrosis after refractive corneal surgery. Ophthalmology, 108, 239-240. http://dx.doi.org/10.1016/S0161-6420(00)00393-6

[3] Chou, T.-C. (2006) Theoretical basis, experimental design, and computerized simulation of synergism and antagonism in drug combination studies. Pharmacological Reviews, 58, 621-681. http://dx.doi.org/10.1124/pr.58.3.10

[4] Sriram, S., Schultz, G.S., Lewin, A., Robinson, P.M., Gibson, D.J. and Tuli, S.S. (2012) Combination siRNA Treatment to Knockdown CTGF, TGF-ss1, TGF-ssR2 and Collagen in Rabbit Corneal Fibroblasts. ARVO Meeting Abstracts, 53, 2209.

[5] Hao, J., Li, S.K., Kao, W.W.Y. and Liu, C.-Y. (2010) Gene delivery to cornea. Brain Research Bulletin, 81, 256-261. http://dx.doi.org/10.1016/j.brainresbull.2009.06.011

[6] Lee, V.H. and Robinson, J.R. (1986) Topical ocular drug delivery: Recent developments and future challenges. Journal of Ocular Pharmacology, 2, 67-108. http://dx.doi.org/10.1089/jop.1986.2.67

[7] La Fuente de, M., Seijo, B. and Alonso, M.J. (2008) "Bioadhesive hyaluronan-chitosan nanoparticles can transport genes across the ocular mucosa and transfect ocular tissue. Gene Therapy, 15, 668-676. http://dx.doi.org/10.1038/gt.2008.16

[8] Sriram, S., Robinson, P., Lewin, A. and Schultz, G. (2013) Nanoparticle vectored siRNAs reduce profibrotic gene expression in wounded rabbit corneas. ARVO Meeting Abstracts, 54, 3878.
[9] Netto, M.V., Mohan, R.R., Sinha, S., Sharma, A., Dupps, W. and Wilson, S.E. (2006) Stromal haze, myofibroblasts, and surface irregularity after PRK. Experimental Eye Research, 82, 788-797. http://dx.doi.org/10.1016/j.exer.2005.09.021

[10] Rodriguez-Lanetty, M. (2007) Trizol/RNeasy hybrid RNA extraction protocol.

[11] Massague, J. (1990) The transforming growth factor-beta family. Annual Review of Cell Biology, 6, 597-641. http://dx.doi.org/10.1146/annurev.cb.06.110190.003121

[12] Grotendorst, G.R. (1997) Connective tissue growth factor: A mediator of TGF-beta action on fibroblasts. Cytokine Growth Factor Reviews, 8, 171-179. http://dx.doi.org/10.1016/S1359-6101(97)00010-5

[13] Jester, J.V., Petroll, W.M. and Cavanagh, H.D. (1999) Corneal stromal wound healing in refractive surgery: The role of myofibroblasts. Progress in Retinal and Eye Research, 18, 311-356. http://dx.doi.org/10.1016/S1350-9462(98)00021-4

[14] Desmoulière, A., Geinoz, A., Gabbiani, F. and Gabbiani, G. (1993) Transforming growth factor-beta 1 induces alphasmooth muscle actin expression in granulation tissue myofibroblasts and in quiescent and growing cultured fibroblasts. The Journal of Cell Biology, 122, 103-111. http://dx.doi.org/10.1083/jcb.122.1.103

[15] Tani, E., Katakami, C. and Negi, A. (2002) Effects of various eye drops on corneal wound healing after superficial keratectomy in rabbits. Nippon Ganka Gakkai Zasshi, 106, 135-142.

[16] Mohan, R.R., Tovey, J.C.K., Sharma, A. and Tandon, A. (2012) Gene therapy in the cornea: 2005-present. Progress in Retinal and Eye Research, 31, 43-64. http://dx.doi.org/10.1016/j.preteyeres.2011.09.001

[17] Hao, J., Li, S.K., Liu, C.-Y. and Kao, W.W.Y. (2009) Electrically assisted delivery of macromolecules into the corneal epithelium. Experimental Eye Research, 89, 934-941. http://dx.doi.org/10.1016/i.exer.2009.08.001 


\section{APPENDIX}

Table A1. TAQMANTM RT PCR primers and probe sequences.

\begin{tabular}{ccc}
\hline Growth Factor & Species & Accession Number \\
\hline \multirow{3}{*}{ CTGF } & Forward & AGGAGTGGGTGTGTGATGAG \\
& Reverse & CCAAATGTGTCTTCCAGTG \\
& Probe & ACCACACCGTGGTTGGCCC \\
TGFB1 & Forward & CCTGTACAACCAGCACAACC \\
& Reverse & CGTAGTACACGATGGGCAGT \\
& Probe & CTCCAGCGCCTGTGGCACAC \\
GAPDH & Forward & GAGACACGATGGTGAAGGTC \\
& Reverse & ACAACATCCACTTTCCAGA \\
SMA & Frobe & CCAATGCGGCCAAATCCGTT \\
& Forward & AGAGCGCAAATACTCCGTCT \\
& Reverse & CCTGTTTGCTGATCCACATC \\
& Probe & CGGCTCCATCCTGGCCTCTC \\
18S rRNA & Forward & GCCGCTAGAGGTGAAATTCTTG \\
& Reverse & CATTCTTGGCAAATGCTTTCG \\
& Probe & ACCGGCGCAAGACGGACCAG \\
\hline
\end{tabular}

